\title{
ESTUDO FITOQUíMICO E AVALIAÇÃO DA ATIVIDADE MOLUSCICIDA DO Calophyllum brasiliense CAMB (CLUSIACEAE)
}

Arquimedes Gasparotto Jr., Mislaine Adriana Brenzan, Izabel Cristina Piloto e Diógenes Aparício Garcia Cortez*

Departamento de Farmácia e Farmacologia, Universidade Estadual de Maringá, Av. Colombo, 5790, 87020-900 Maringá - PR Celso Vataru Nakamura e Benedito Prado Dias Filho

Departamento de Análises Clínicas, Universidade Estadual de Maringá, Av. Colombo, 5790, 87020-900 Maringá - PR

Edson Rodrigues Filho e Antonio Gilberto Ferreira

Departamento de Química, Universidade Federal de São Carlos, CP 676, 13560-970 São Carlos - SP

Recebido em 5/1/04; aceito em 17/12/04; publicado na web em 28/2/05

PHYTOCHEMICAL STUDY AND EVALUATION OF THE MOLLUSCICIDAL ACTIVITY OF Calophyllum brasiliense CAMB (CLUSIACEAE). The bioassay-guided fractionation against Biomphalaria glabrata of hydroalcoholic extracts of Calophyllum brasiliense aerial parts led to the isolation of the coumarin, named (-) mammea A/BB. The compound had its structure determined by both spectroscopic techniques (NMR ${ }^{1} \mathrm{H}$, NMR ${ }^{13} \mathrm{C}$, gHSQC, gHMBC and MS) and some literature comparison data. The probit analysis of (-) mammea $\mathrm{A} / \mathrm{BB}$ showed $\mathrm{LD}_{50}=0.67 \mathrm{ppm}$ and $\mathrm{LD}_{90}=1.47 \mathrm{ppm}$. In addition, the dichloromethane extract obtained from C. brasiliense leaves with significant molluscicidal activity against Biomphalaria glabrata was analyzed by HPLC-UV.

Keywords: Calophyllum brasiliense; molluscicidal activity; coumarin.

\section{INTRODUÇÃO}

O Calophyllum brasiliense Camb. é uma árvore de grande porte, que cresce principalmente em regiões de floresta da Mata Atlântica no Brasil. Regionalmente conhecido como guanandi, o chá de suas cascas obtido por infusão é um remédio popular utilizado para tratamento de reumatismo, varicoses, hemorróidas e úlceras crônicas $^{1}$. Em 1992, um grupo de pesquisadores do Instituto Nacional do Câncer reportou uma cumarina, o (+) calanolideo A, isolada de espécies de Calophyllum, como uma das substâncias químicas mais ativas frente ao vírus HIV-12. Estudos fitoquímicos do caule e da resina do Calophyllum brasiliense revelaram a presença de diversas substâncias químicas, tais como xantonas, sitosterol e triterpenos ${ }^{3}$. Algumas cumarinas isoladas desta espécie apresentaram atividade anticancerígena e antimicrobiana ${ }^{2,4}$. O extrato em diclorometano do caule $C$. brasiliense apresentou um efeito gastroprotetor em ratos ${ }^{5}$.

Extratos brutos obtidos de diversas partes de espécies da família Clusiaceae, como Calophyllum verticillatum, C. inophyllum e C. Recedens, apresentaram atividade moluscicida na concentração de $100 \mathrm{ppm}$ para a primeira espécie e $10 \mathrm{ppm}$ para as outras duas ${ }^{6}$. A cumarina $( \pm)$ mammea $\mathrm{A} / \mathrm{BB}$, isolada das sementes do $C$. Verticillatum, apresentou atividade moluscicida na concentração de 10 ppm, com $20 \%$ de mortalidade dos moluscos B. glabrata ${ }^{7}$. O extrato metanólico do caule de Kielmeyera variabilis apresentou forte atividade moluscicida em $12,5 \mathrm{ppm}^{8}$.

As substâncias moluscicidas são um fator crucial para o controle da esquistossomose. No momento, apenas uma substância sintética, a niclosamida, é recomendada pela ONU como moluscicida ${ }^{9}$. Em países do terceiro mundo, o uso de moluscicidas sintéticos tem causado problemas de toxicidade, contaminação do meio ambiente e resistência dos caramujos (Biomphalaria glabrata) transmissores da esquistossomose. Em contraste, o uso de plantas com atividade moluscicida pode representar uma alternativa barata, além de evitar a poluição do meio ambiente, pelo fato de elas serem biodegradáveis ${ }^{10}$.

Assim, o presente trabalho tem como objetivo isolar o(s) princípio(s) ativo(s) das partes aéreas do Calophyllum brasiliense Camb., responsável pela atividade moluscicida.

\section{RESULTADOS E DISCUSSÃO}

Os extratos brutos obtidos das folhas e galhos de $C$. brasiliense foram submetidos ao bioensaio de atividade moluscicida com Biomphalaria glabrata, sendo que o extrato RF foi ativo na concentração de 25 ppm, com 100\% de mortalidade. Esse extrato foi refracionado e apenas a fração $\mathbf{F 1}$ apresentou atividade em 1,20 ppm, com $100 \%$ de mortalidade (Tabela 1). A fração F1 resultou no isolamento de uma cumariana denominada de (-) mammea A/ BB (1). A substância 1 apresentou após 24 h uma $\mathrm{DL}_{50}$ de 0,67 ppm e $\mathrm{DL}_{90}$ de 1,47 ppm (Tabela 2). Por meio dos dados parciais apresentados, pode-se concluir que a substância 1 tem potencial moluscicida, quando comparada com a niclosamida $\left(\mathrm{DL}_{50}\right.$ de $0,77 \mathrm{ppm}$ e $\mathrm{DL}_{90}$ de $\left.1,75 \mathrm{ppm}\right)^{11}$, podendo ser um substituto em potencial desta substância que é utilizada atualmente. Estes resultados diferem dos obtidos pela literatura para a $( \pm)$ mammea $\mathrm{A} / \mathrm{BB}$ isolada das se-<smiles>CC[C@H](C)C(=O)c1c(O)c(CC=C(C)C)c(O)c2c(-c3ccccc3)cc(=O)oc12</smiles>

1 
Tabela 1. Atividade moluscicida sobre a Biomphalaria glabrata dos extratos RF, LF, RG e LG das frações $\mathbf{F 1}$ a $\mathbf{F 8}$ e da substância química 1 (subfração F1.12) do Calophyllum brasiliense e do moluscicida sintético niclosamida, que foi utilizado como controle positivo (C)

\begin{tabular}{cccccccccccccccccccccc}
\hline $\begin{array}{l}\text { Tempo de } \\
\text { exposição }\end{array}$ & $6 \mathrm{~h}$ & $24 \mathrm{~h}$ & $6 \mathrm{~h}$ & $24 \mathrm{~h}$ & $6 \mathrm{~h}$ & $24 \mathrm{~h}$ & $6 \mathrm{~h}$ & $24 \mathrm{~h}$ & $6 \mathrm{~h}$ & $24 \mathrm{~h}$ & $6 \mathrm{~h}$ & $24 \mathrm{~h}$ & $6 \mathrm{~h}$ & $24 \mathrm{~h}$ & $6 \mathrm{~h}$ & $24 \mathrm{~h}$ & $6 \mathrm{~h}$ & $24 \mathrm{~h}$ \\
\hline [ ]ppm & 100 & 100 & 50 & 50 & 25 & 25 & 10 & 10 & 5,0 & 5,0 & 2,5 & 2,5 & 1,2 & 1,2 & 0,6 & 0,6 & 0,3 & 0,3 \\
\hline RF & - & + & - & + & - & + & - & - & - & - & - & - & - & - & - & - & - & - \\
LF & - & + & - & - & - & - & - & - & - & - & - & - & - & - & - & - & - & - \\
RG & - & + & - & - & - & - & - & - & - & - & - & - & - & - & - & - & - & - \\
LG & - & + & - & - & - & - & - & - & - & - & - & - & - & - & - & - & - & - \\
F1 & - & + & - & + & - & + & - & + & - & + & - & + & - & + & - & - & - & - \\
F2 & - & - & - & - & - & - & - & - & - & - & - & - & - & - & - & - & - & - \\
F3 & - & - & - & - & - & - & - & - & - & - & - & - & - & - & - & - & - & - \\
F4 & - & - & - & - & - & - & - & - & - & - & - & - & - & - & - & - & - & - \\
F5 & - & - & - & - & - & - & - & - & - & - & - & - & - & - & - & - & - & - \\
F6 & - & - & - & - & - & - & - & - & - & - & - & - & - & - & - & - & - & - \\
F7 & - & - & - & - & - & - & - & - & - & - & - & - & - & - & - & - & - & - \\
F8 & - & - & - & - & - & - & - & - & - & - & - & - & - & - & - & - & - & - \\
F9 & - & - & - & - & - & - & - & - & - & - & - & - & - & - & - & - & - & - \\
C & + & + & + & + & + & + & + & + & + & + & + & + & + & + & + & + & + & + \\
\hline
\end{tabular}

$\mathbf{L F}=$ extrato liofilizado das folhas; $\mathbf{L G}=$ extrato liofilizado dos galhos; $\mathbf{R F}=$ resíduo das folhas, $\mathbf{R G}=$ resíduo dos galhos; $(+)=$ caramujos mortos; $(-)$ caramujos sobreviventes.

Tabela 2. Valores de $\mathrm{DL}_{50}$ e $\mathrm{DL}_{90}(\mathrm{ppm})$ e intervalos de confiança de $95 \%$ para (-) mammea $\mathrm{A} / \mathrm{BB}(\mathbf{1})$

\begin{tabular}{ccccc}
\hline Tempo de exposição & $\mathrm{DL}_{50}$ & Intervalo de confiança & $\mathrm{DL}_{90}$ & Intervalo de confiança \\
\hline $24 \mathrm{~h}$ & 0,67 & $0,48-0,93$ & 1,47 & $1,00-2,99$ \\
\hline
\end{tabular}

mentes do C. verticillatum, que apresentaram atividade em $10 \mathrm{ppm}$, com $20 \%$ de mortalidade dos caramujos B. glabrata ${ }^{7}$. A substância 1 isolada das folhas do $C$. brasiliense, com uma rotação óptica $[\alpha]_{\mathrm{D}}=-10\left(\mathrm{c} 0,14, \mathrm{CHCl}_{3}\right)$, apresentou maior atividade que sua forma racêmica, isolada do $C$. verticillatum, podendo-se supor que o enantiômero levógiro é mais ativo que a mistura racêmica.

Para confirmar a fórmula molecular $\mathrm{C}_{25} \mathrm{H}_{26} \mathrm{O}_{5}$ e a massa molecular de 406 u.m.a. da estrutura proposta para $\mathbf{1}$, foi obtido o espectro de massas dessa substância, utilizando a ionização por "electrospray" (ESI-MS), em que foram observados o íon quasi molecular [M-H]'em m/z 405 (24\%), compatível com a estrutura proposta. $\mathrm{O}$ espectro de $\mathrm{RMN}{ }^{1} \mathrm{H}$ mostrou sinais de quatro metilas H-4": $\delta_{\mathrm{H}} 1,70 \mathrm{sl} ; \mathrm{H}^{\prime \prime}$ " $^{\prime} \delta_{\mathrm{H}} 1,65 \mathrm{~d}, \mathrm{~J}=1,2 \mathrm{~Hz} ; \mathrm{H}-3^{\prime \prime \prime}: \delta_{\mathrm{H}} 1,28 \mathrm{~d}$, $\mathrm{J}=6,9 \mathrm{~Hz}$ e H-5"'”: $\delta_{\mathrm{H}} 0,99 \mathrm{t}=6,6 \mathrm{~Hz}$ ), dois hidrogênios olefínicos, um multipleto em $\delta_{\mathrm{H}} 5,07-5,12(\mathrm{~m})$ e um singleto em $\delta_{\mathrm{H}} 6,00$, representantes dos hidrogênios $\mathrm{H}-2$ " e $\mathrm{H}-3$, respectivamente, e dois sinais de hidrogênios metilênicos $\mathrm{H}^{-1} 1^{\prime \prime}: \delta_{\mathrm{H}} 3,29 \mathrm{~d}, \mathrm{~J}=6,9 \mathrm{~Hz}$ e H $\mathrm{A}^{-}$ 4"”: $\delta_{\mathrm{H}} 1.81-2,00 \mathrm{~m} ; \mathrm{H}_{\mathrm{B}}-4^{\prime \prime \prime}: \delta_{\mathrm{H}} 1,42-1,52 \mathrm{~m}$. A presença de um anel fenila foi reconhecida pela presença de dois multipletos em $\delta_{\mathrm{H}}$ 7,41-7,44 e $\delta_{\mathrm{H}} 7,53-7,58 \mathrm{com}$ uma integral para cinco hidrogênios. No espectro de $\mathrm{RMN}{ }^{1} \mathrm{H}$, foi observado na região desblindada um singleto em $\delta_{\mathrm{H}} 14,57$ referente a uma hidroxila quelatogênica. A posição do grupamento prenila no carbono C-6 foi determinada através das interações heteronucleares de átomos de carbono e hidrogênio observados nos espectros gHSQC $\left({ }^{1} \mathrm{~J}_{\mathrm{CH}}\right) \operatorname{gHMBC}\left({ }^{2,3} \mathrm{~J}_{\mathrm{CH}}\right)$, permitindo também identificar os sinais dos carbonos hidrogenados e quaternários da unidade aromática (Tabela 3). Os dados espectroscópicos da substância isolada foram comparados aos da literatura para a ( \pm ) mammea $\mathrm{A} / \mathrm{BB}^{4,12}$. Com base nesta comparação, a substância isolada foi caracterizada como (-) mammea A/BB (1).

No cromatograma típico do extrato em diclorometano $(\mathbf{R F})$ das folhas de $C$. brasiliense, apresentado na Figura 1, foi possível identificar a substância 1 com o tempo de retenção de 28,84 min, por meio da comparação dos dados da literatura de ultravioleta obtidos pelo detector de arranjo de fotodiodo e por comparação com o cromatograma da amostra pura, realizados nas mesmas condições. O perfil cromatográfico obtido do extrato RF permite sua padronização, para fins de controle de qualidade, contribuindo para a futura utilização desse extrato bruto no controle da esquistossomose como alternativa à utilização da substância 1 .

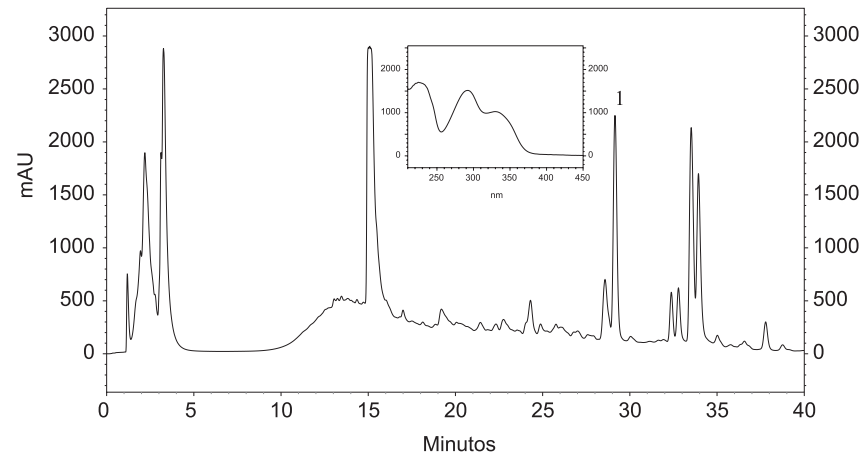

Figura 1. Cromatograma típico a $254 \mathrm{~nm}$ do extrato em diclorometano $(\boldsymbol{R} \boldsymbol{F})$ das folhas de C. brasiliense em CLAE com coluna Metasil ODS. Fase móvel 0,6 mL/min. ACN-H2O 5:95 - 55:45 em $10 \mathrm{~min}$., 55:45 - 80:20 em $20 \mathrm{~min}$, $80: 20$ - $100 \mathrm{em} 30$ min e $100 \mathrm{em} 40 \mathrm{~min}$. Detector arranjo de fotodiodo ( $\lambda$ : 200-450 nm, monitorado em m $254 \mathrm{~nm}$ ). (1): (-) mammea A/BB

\section{PARTE EXPERIMENTAL}

\section{Procedimentos experimentais gerais}

Os espectros no infravermelho foram registrados em espectrômetro Bomen - MV 100, Hartmann \& Braun- Michelson. Os espectros de $\mathrm{RMN}$ de ${ }^{1} \mathrm{H}(400,13 \mathrm{MHz})$ e de ${ }^{13} \mathrm{C}(100,6 \mathrm{MHz})$ foram obtidos em espectrômetro Bruker Avance e Varian, modelos 
Tabela 3. Dados de $\mathrm{RMN}^{1} \mathrm{H}\left(\delta, \mathrm{CDCl}_{3}, 300 \mathrm{MHz}\right)$ e $\mathrm{RMN}^{13} \mathrm{C}(100$ $\mathrm{MHz})$ de $1^{*}$

\begin{tabular}{|c|c|c|c|}
\hline \multirow[b]{3}{*}{$\mathrm{C}$} & \multicolumn{3}{|c|}{1} \\
\hline & & gHSQC & gHMBC \\
\hline & & $\delta_{\mathrm{H}}$ & \\
\hline 2 & 158,6 & & H-3 \\
\hline 3 & 112,1 & $6,00(\mathrm{~s})$ & \\
\hline 4 & 154,2 & & \\
\hline $4 \mathrm{a}$ & 100,5 & & \\
\hline 5 & 157,1 & & H-6',H-2',H-1'”,H-3 \\
\hline 6 & 112,6 & & \\
\hline 7 & 166,9 & & $\mathrm{H}-1 ”$ \\
\hline $7-\mathrm{OH}$ & & $14,57(\mathrm{~s})$ & \\
\hline 8 & 104,2 & & $\mathrm{H}-3$ \\
\hline $8 a$ & 155,7 & & $\mathrm{H}-3$ \\
\hline 1 ' & 136,8 & & \\
\hline 2 ' & 127,5 & 7,41-7,44 (m) & \\
\hline 3 ' & 129,5 & $7,53-7,58(\mathrm{~m})$ & H-2',H-6' \\
\hline 4, & 130,2 & $7,53-7,58(\mathrm{~m})$ & \\
\hline 5 , & 129,5 & 7,53-7,58 (m) & H-2',H-6' \\
\hline 6, & 127,5 & $7,41-7,44(\mathrm{~m})$ & \\
\hline $1 "$ & 21,6 & 3,29-(d, J=6,9 Hz) & \\
\hline $2 "$ & 120,8 & $5,07-5,12(\mathrm{~m})$ & H-1",H-4”,H-5" \\
\hline $3 "$ & 134,1 & & H-1",H-4”,H-5" \\
\hline $4 "$ & 17,8 & $1,70(\mathrm{sl})$ & \\
\hline $5 "$ & 25,7 & $1,65(\mathrm{~d}, \mathrm{~J}=1,2 \mathrm{~Hz})$ & \\
\hline $1, "$ & 210,5 & & $\begin{array}{l}\text { H-2,",,HA-4,", ,HB-4,", } \\
\text { H-5,", }\end{array}$ \\
\hline $2 "$, & 47,0 & $3,96(\operatorname{sex} \mathrm{J}=6,9 \mathrm{~Hz})$ & HA-4",',HB-4",', H-5,", \\
\hline $3^{\prime \prime \prime}$ & 16,6 & $1,28(\mathrm{~d}, \mathrm{~J}=6,9 \mathrm{~Hz})$ & $\begin{array}{l}\text { HA-4,",HB-4",', H-2,", } \\
\text { H-3,", }\end{array}$ \\
\hline A $4, "$, & 27,2 & $1,81-2,00(\mathrm{~m})$ & H-5",', H-2",' H-3", \\
\hline B4,", & & $1,42-1,52(\mathrm{~m})$ & \\
\hline $5^{\prime \prime}$ & 11,8 & $0,99(\mathrm{t}=6,6 \mathrm{~Hz})$ & \\
\hline
\end{tabular}

*Os sinais de carbonos $\mathrm{C}, \mathrm{CH}$, e $\mathrm{CH}_{3}$ foram reconhecidos pela análise comparativa dos espectros de DEPT $90^{\circ}$ e $135^{\circ}$. O espectro de $2 \mathrm{D}$ gCOSY foi utilizado, os deslocamentos químicos e constantes de acoplamentos (J) dos sinais de hidrogênio foram deduzidos do espectro 1D de $\mathrm{RMN}^{1} \mathrm{H}$.

Gemini 2000 BB, $300 \mathrm{MHz}\left(300,06 \mathrm{MHz}\right.$ para ${ }^{1} \mathrm{H}$ e 75,45 MHz para ${ }^{13} \mathrm{C}$ ), utilizando-se $\mathrm{CDCl}_{3}$ como solvente e TMS como referência interna. Para obtenção de espectro no ultravioleta foi utilizado o aparelho Varian Cary 1E. Os espectros de massa por "electrospray" foram obtidos em espectrômetro Micromass Quattro LC. O espectro de CD foi obtido no equipamento Jasco 810.

As separações cromatográficas em coluna por adsorção foram realizadas utilizando-se a técnica de cromatografia no modo "flash" com sílica-gel 60 (230-400 mesh ASTM, Merck). Para as cromatografias em camada delgada analítica utilizou-se sílica-gel $\mathrm{GF}_{254}$ Merck, e as placas foram reveladas sob luz ultravioleta, nos comprimentos de onda de 254 a 366 nm, e pela utilização de reveladores com vanilina-ácido sulfúrico e vapores de iodo. Foram também utilizadas CC em gel de SEPHADEX ${ }^{\circledR}$ LH-20 para a purificação de substâncias.

\section{Material vegetal}

As partes aéreas foram coletadas na Ilha do Cardoso no estado de São Paulo, e uma exsicata foi depositada no herbário do Instituto de Botânica de São Paulo (SP 363818).

\section{Obtenção dos extratos}

As folhas e galhos, depois de secos em temperatura ambiente, foram triturados em moinho de facas e martelo, obtendo-se as massas do pó de 985 e 446 g, respectivamente O pó dos galhos e das folhas foi extraído pelo processo de maceração com etanol:água (9:1) até o esgotamento total dos princípios ativos. Os extratos hidroalcoólicos das folhas e galhos foram filtrados e evaporados em rotaevaporador a vácuo em temperatura de $45{ }^{\circ} \mathrm{C}$, até a eliminação total do solvente orgânico. Do concentrado, em forma de xarope viscoso das folhas e caules, foram separados os resíduos e solubilizados em diclorometano e denominados $\mathbf{R F}=$ resíduo das folhas e $\mathbf{R G}$ = resíduo dos galhos. O solvente orgânico foi eliminado em rotaevaporador à vácuo, a $45^{\circ} \mathrm{C}$. Os filtrados das folhas e caules foram liofilizados e armazenados em freezer e denominados $\mathbf{L F}=$ liofilizado das folhas e $\mathbf{L G}=$ liofilizado dos galhos. Foram obtidos os extratos brutos das folhas $\mathbf{R F}=30,87 \mathrm{~g}$ e $\mathbf{L F}=$ $150 \mathrm{~g}$ e dos galhos $\mathbf{R G}=2,94 \mathrm{~g}$ e $\mathbf{L G}=41,5 \mathrm{~g}$.

\section{Extração e fracionamento}

O extrato bruto das folhas em diclorometano $(\mathbf{R F}=23,54 \mathrm{~g})$ foi cromatografado em coluna empacotada com sílica-gel à vácuo, utilizando-se como fase móvel solventes orgânicos de polaridade crescente: hexano; hexano:diclorometano (1:1); diclorometano; diclorometano:acetato de etila (9:1); diclorometano:acetato de etila (4:1); diclorometano:acetato de etila (1:1); acetato de etila; metanol, e metanol:água (9:1). Foram obtidas 9 frações após a evaporação do solvente orgânico: $(\mathbf{F 1}=8,73 \mathrm{~g}),(\mathbf{F} 2=1,82 \mathrm{~g}),(\mathbf{F 3}=0,63 \mathrm{~g})$, $(\mathbf{F} 4=0,88 \mathrm{~g}),(\mathbf{F 5}=0,61 \mathrm{~g}),(\mathbf{F 6}=1,12 \mathrm{~g}),(\mathbf{F} 7=2,26 \mathrm{~g}),(\mathbf{F 8}=$ $5,47 \mathrm{~g})$ e $(\mathbf{F 9}=0,09 \mathrm{~g})$. As frações obtidas foram submetidas a ensaio da atividade moluscicida, utilizando-se caramujos da espécie Biomphalaria glabrata (Tabela 1). A fração bioativa F1 (5,0 g) foi cromatografada em coluna de sílica-gel, utilizando-se como eluentes: hexano; hexano:diclorometano (98:2, 95:5, 90:10, 80:20 e 50:50); diclorometano; diclorometano:acetato de etila (98:2, 95:5, 90:10, 80:20 e 50:50); acetato de etila, e metanol, sendo obtidas 122 frações. As frações F9 a F12 e F73 a F77 com identidade cromatográfica foram reunidas, obtendo-se as subfrações $\mathbf{F 1 . 1 2}$ (58,2 mg) e F1.77 (278 mg), as quais foram submetidas à atividade moluscicida (Tabela 1). A subfração F1.12 foi identificada como uma cumarina, a (-) mammea A/BB (1) e a subfração F1.77 como uma mistura de três xantonas, por meio da análise dos espectros de UV, IV, EM. Foi feita aplicação de técnicas bidimensionais de RMN (gCOSY, gHSQC, gHMBC), comparando-se com os dados registrados na literatura ${ }^{4,12}$.

Mammea A/BB (1). Cristal branco, ponto de fusão $124-125^{\circ} \mathrm{C}$ (lit.:124-125 ${ }^{\circ} \mathrm{C}$, hexano) ${ }^{13} ;[\alpha]_{\mathrm{D}}=-10\left(\mathrm{CHCl}_{3}, \mathrm{c} 0,14\right), \mathrm{CD}_{\lambda \max } \mathrm{nm}$ $(\Delta \varepsilon): 287(+3,69), 316(-3,23), \mathrm{UV}\left(\mathrm{CHCl}_{3}\right) \lambda_{\max } / \mathrm{nm}(\log \varepsilon): 226$ (4,42), 295 (4,28), 333 (4,24). IV: nmáx. ${ }^{\mathrm{NaCl}} \mathrm{cm}^{-1}: 3439,2926,1733$, 1596, 1441, 1391, 1229, 1185, 1095, 774. RMN de ${ }^{1} \mathrm{H}$ (Tabela 2). RMN de ${ }^{13} \mathrm{C}$ (Tabela 2). ESI-MS: $m / z$ (int. rel.): 405 [M-H] ${ }^{-}(100)$. ESI-MS/MS: $m / z$ (int. rel.): 405 [M-H] $^{-}$(24), 277(100), 305 (51), 333 (36).

\section{ENSAIO BIOLÓGICO}

\section{Avaliação da atividade moluscicida ${ }^{14}$}

O ensaio moluscicida foi realizado com os extratos $\mathbf{R F}, \mathbf{L F}$, RG e LG, frações F1 a F8, subfração F1.77 e substância química 1. Para cada concentração foram utilizados três caramujos da espécie Biomphalaria glabrata de tamanho uniforme. As amostras fo- 
ram diluídas em água filtrada do aquário, sem cloro, com o auxílio de $100 \mu \mathrm{l}$ de DMSO nas concentrações de $100 ; 50 ; 25 ; 10 ; 5,0 ; 2,5$; 1,2; 0,6 e 0,3 ppm em temperatura ambiente. Cada caramujo ficou separadamente, em contato com $50 \mathrm{ml}$ dessa solução. Foi utilizada uma prova em branco apenas com o DMSO e como controle positivo a niclosamida. Foram realizadas leituras em 6 e 24 h e após esse tempo, foram observados os batimentos cardíacos por meio de uma lupa para verificar a mortalidade deles.

Para a obtenção de $\mathrm{DL}_{50}$ e $\mathrm{DL}_{90}$ da substância pura, foram utilizados dez caramujos adultos da espécie B. glabrata para cada concentração da amostra, de acordo com as recomendações da Organização Mundial da Saúde ${ }^{15}$ para a análise de plantas moluscicidas.

Os dados obtidos foram analisados pelo Programa Probit Versão $1.5^{16}$.

\section{AGRADECIMENTOS}

Ao CNPq e CAPES pelas bolsas e apoio financeiro aos Profs. D. A. G. Cortez e B. P. Dias Filho; a aluna de Iniciação Científica M. A. Brenzan; à Profa ${ }^{a}$ M. C. M. Young do Instituto de Botânica de São Paulo, pela coleta e identificação botânica do espécimen utilizado e ao Prof. R. J. Ward, do Departamento de Química da FFCLRP-USP, pela obtenção do espectro de CD.

\section{REFERÊNCIAS}

1. Correa, M. P.; Dicionário das Plantas Úteis do Brasil e das Exóticas Cultivadas, Imprensa Nacional: Rio de Janeiro, 1984, vol. III, p. 388.

2. Ito, C.; Itoigawa, M.; Mishina, Y.; Cechinel-Filho, V.; Enjo, E.; Tokuda, H.; Nishino, H.; Furukawa.; J. Nat. Prod. 2003, 66, 368.

3. Da Silva, K. L.; Dos Santos, A. R.; Mattos, P. E.; Yunes, R. A.; DelleManache, F.; Cechiinel-Filho, V.; Therapie 2001, 56, 431.

4. Chilpa, R. R.; Muñiz, E. E.; Apan, T. R.; Amekraz, B.; Aumelas, A.; Jankowski. K.; Torrez, M. V.; Life Sci. 2004, 75, 1635.

5. Sartori, N. T.; Canepelle, D.; Sousa Jr, P. T.; Martins, D. T. O.; J. Ethnopharmacol. 1999, 67, 149.

6. Ravelonjato, B.; Kunesch, N.; Poisson, J. E.; Phytochemistry 1987, 26, 2973.

7. Ravelonjato, B.; Libot, F.; Ramiandrasoa, F.; Kunesch, N.; Cayral, P.; Poisson, J.; Planta Med. 1992, 58, 51.

8. Pinheiro, L.; Vidotti, G. J.; Young, M. C. M.; Ferreia, A. G.; Cortez, D. A. G.; Quim. Nova 2003, 26, 157.

9. D'Arcy, P.; Harron, D. W. G.; Pharm. Int. 1983, 4, 16.

10. Marston, A.; Hostettmann, K.; Phytochemistry 1985, 24, 639.

11. Giovanelli, A.; Silva, C. L. P. A.; Medeiros, L.; Vasconcellos, M. C.; Mem. Inst. Oswaldo Cruz 2002, 97, 743.

12. Morel, C.; Guilet, D.; Oger, J. M.; Séraphin, D.; Sévenet, T.; Wiart, C.; Hamid, A. H. A.; Richomme, P.; Bruneton, J.; Phytochmeistry 1999, 50, 1243.

13. Combrie, L.; Games, D. E.; J. Chem. Soc. (C) 1967, 2553.

14. Hostettmann, H.; Kizu, H.; Tomimori, T.; Planta Med. 1982, 44, 34.

15. WHO-World Health Organization; Bull. Wld. Hlth. Org. 1984, 33, 567.

16. http://www.epa.gov/nerleerd/stat2.htm\#probit, acessada em Agosto 2004. 